\title{
Avaliação do Índice de Qualidade das águas e da contaminação por tóxicos do Ribeirão do Carmo em Monsenhor Horta, distrito de Mariana - MG
}

\author{
Evaluation of the Water Quality and Toxic Contamination Index of the Ribeirão do Carmo in \\ Monsenhor Horta, Mariana district - MG \\ Evaluación del Índice de Calidad del agua y contaminación tóxica del Ribeirão do Carmo en
}

Monsenhor Horta, distrito de Mariana - MG

Recebido: 24/07/2021 | Revisado: 30/07/2021 | Aceito: 02/08/2021 | Publicado: 06/08/2021

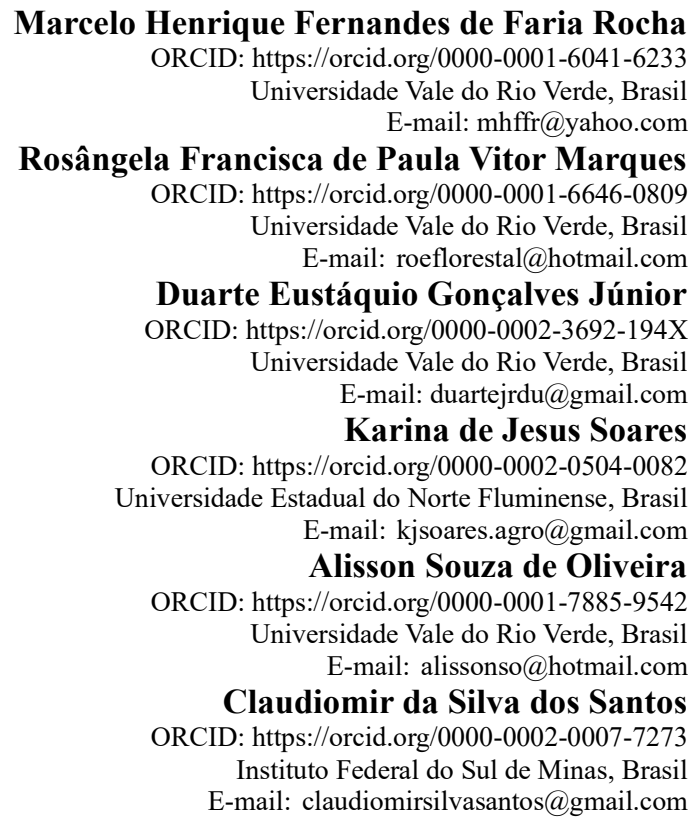

\begin{abstract}
Resumo
O presente trabalho avaliou o Índice de Qualidade das Águas (IQA) e a Contaminação por Tóxicos do Ribeirão do Carmo com base nos resultados obtidos pelo programa de monitoramento de qualidade das águas realizado pelo Instituto Mineiro de Gestão das Águas (IGAM) entre os anos de 2010 e 2019 na estação amostral denominada "RD009", localizada em Monsenhor Horta, distrito do município de Mariana, no estado de Minas Gerais. Foram adotados nove parâmetros mais representativos para a caracterização da qualidade de água e seus respectivos pesos de acordo com sua importância relativa no cálculo do IQA. A contaminação por tóxicos foi classificada em "Baixa", "Média" ou "Alta" a partir das concentrações observadas de quatorze parâmetros tóxicos. Foi realizada a análise temporal para acompanhamento da evolução do IQA e contaminação por tóxicos além de comparativo com os critérios preconizados na Deliberação Normativa COPAM-CERH n 01/2008 para classe 2. Os resultados apontam que o IQA ao longo da série histórica considerada é "médio" enquanto a contaminação por tóxicos foi classificada como "alta". As variáveis coliformes termotolerantes, fósforo total e arsênio total, que apresentaram resultados acima do máximo permitido pela legislação, ao longo de praticamente todo período avaliado, correlacionam-se com as características do uso e ocupação da terra na região. Neste sentido, faz-se necessário que os organismos públicos de gestão e governança de recursos hídricos formulem diretrizes e estratégias de melhoria da qualidade das águas do Ribeirão do Carmo tendo em vista os riscos à saúde da população do distrito de Monsenhor Horta.
\end{abstract}

Palavras-chave: IQA; Coliformes termotolerantes; Fósforo total; Arsênio total; Garimpos de ouro

\begin{abstract}
The present work evaluated the Water Quality Index (IQA) and the Contamination by Toxics of Ribeirão do Carmo based on the results obtained by the water quality monitoring program carried out by the Mineiro Institute of Water Management (IGAM) between the years of 2010 and 2019 at the sampling station named "RD009", located in Monsenhor Horta, district of the municipality of Mariana, in the state of Minas Gerais. Nine most representative
\end{abstract}


parameters were adopted for the characterization of water quality and their respective weights according to their relative importance in the calculation of the IQA. Toxic contamination was classified as "Low", "Medium" or "High" based on the observed concentrations of fourteen toxic parameters. A temporal analysis was performed to monitor the evolution of the IQA and contamination by toxics, as well as a comparison with the criteria recommended in the Normative Deliberation COPAM-CERH No. 01/2008 for class 2. The results show that the IQA along the considered historical series is " medium" while toxic contamination was rated as "high". The variables thermotolerant coliforms, total phosphorus and total arsenic, which presented results above the maximum allowed by legislation, throughout practically the entire period evaluated, are correlated with the characteristics of land use and occupation in the region. In this sense, it is necessary that public bodies for the management and governance of water resources formulate guidelines and strategies to improve the quality of water in the Ribeirão do Carmo, bearing in mind the health risks to the population of the district of Monsenhor Horta.

Keywords: WQI; Thermotolerant coliforms; Total Phosphorus; Total arsenic; Gold-digging.

\section{Resumen}

El presente trabajo evaluó el Índice de Calidad del Agua (IQA) y la Contaminación por Tóxicos de Ribeirão do Carmo en base a los resultados obtenidos por el programa de monitoreo de la calidad del agua realizado por el Instituto Mineiro de Gestión del Agua (IGAM) entre los años 2010 y 2019. en la estación de muestreo denominada "RD009”, ubicada en Monsenhor Horta, distrito del municipio de Mariana, en el estado de Minas Gerais. Se adoptaron nueve parámetros más representativos para la caracterización de la calidad del agua y sus respectivos pesos según su importancia relativa en el cálculo del IQA. La contaminación tóxica se clasificó como "Baja", "Media" o "Alta" según las concentraciones observadas de catorce parámetros tóxicos. Se realizó un análisis temporal para monitorear la evolución del IQA y contaminación por tóxicos, así como una comparación con los criterios recomendados en la Deliberación Normativa COPAM-CERH No. 01/2008 para la clase 2. Los resultados muestran que el IQA en todo el la serie histórica considerada es "media" mientras que la contaminación tóxica se calificó como "alta". Las variables coliformes termotolerantes, fósforo total y arsénico total, que presentaron resultados por encima del máximo permitido por la legislación, durante prácticamente todo el período evaluado, se correlacionan con las características de uso y ocupación del suelo en la región. En este sentido, es necesario que los organismos públicos de gestión y gobernanza de los recursos hídricos formulen pautas y estrategias para mejorar la calidad del agua en el Ribeirão do Carmo, teniendo en cuenta los riesgos para la salud de la población del distrito de Monsenhor Horta.

Palabras clave IQA; Coliformes termotolerantes; Fósforo total; Arsénico total; Lavado de oro.

\section{Introdução}

Várias são as modalidades de uso e ocupação do solo potencialmente degradantes da qualidade dos recursos hídricos (Araújo et al., 2018).

Seja em zonas urbanas ou rurais, o esgotamento doméstico, fonte de nutrientes (sobretudo de nitrogênio e fósforo) e de inúmeros tipos de microrganismos eliminados nos excrementos humanos (especialmente do grupo coliformes), quando lançado num dado corpo hídrico sem o devido tratamento, implica tanto na degradação da qualidade da água em função da drástica redução dos níveis de oxigênio dissolvido (que acarreta na eutrofização de corpos d'água) quanto na difusão de doenças de veiculação hídrica, quais sejam: ancilostomíase, ascaridíase, amebíase, cólera, diarreia infecciosa, disenteria bacilar, esquistossomose, estrongiloidíase, febre tifoide, febre paratifoide, salmonelose, teníase e cisticercose, dentre outras (Funasa, 2015). Tendo em vista a conhecida e sensível correlação entre saneamento, saúde pública e os ecossistemas, o cenário ora descortinado, recorrente na maioria das cidades brasileiras, culmina no aumento dos custos de tratamento da água e dos dispêndios com diligências hospitalares, revertendo-se, portanto, em severo prejuízo para a sociedade (Funasa, 2015).

Atividades agrícolas que indispõe de mecanismos de controle ambiental podem lançar águas residuárias com elevadas cargas de nutrientes em corpos d'água. É verdade que o nitrogênio e fósforo são insumos estratégicos ao desenvolvimento de culturas nas escalas demandadas pelo mercado consumidor (FAO, 2018). Entretanto, a utilização indiscriminada destes nutrientes pode levar à poluição dos recursos hídricos pelo escoamento superficial, que cria um caminho direto para nutrientes solúveis e sedimentáveis chegarem mais rapidamente aos rios, especialmente em regiões onde inexiste cobertura vegetal (Blann et al., 2009; Kemerich et al., 2014; Rodrigues et al., 2018). Por sua vez, a pecuária ancorada em técnicas de manejo ambientalmente insustentáveis pode ser um fator de deposição de efluentes de curral in natura nos seguimentos fluviais, o que 
Research, Society and Development, v. 10, n. 10, e110101018680, 2021

(CC BY 4.0) | ISSN 2525-3409 | DOI: http://dx.doi.org/10.33448/rsd-v10i10.18680

acarreta tanto no incremento de nitrogênio e fósforo nos rios, que pode levar à eutrofização de corpos d'água, quanto na disseminação de doenças de veiculação hídrica porquanto as fezes animais, a exemplo dos dejetos humanos, são ricas em patógenos (Funasa, 2015).

Inobstante a atividade da mineração artesanal (garimpos) ser até mesmo a única alternativa laboral em localidades desprovidas de pujança socioeconômica, as técnicas empregadas na explotação do ouro são rudimentares (Sobreira, 2014). Os impactos ambientais gerados por esta atividade envolvem a descaracterização da fitofisionomia local em função da supressão vegetal e a descaracterização morfológica do terreno provocada pelo desmonte de encostas com jato hidráulico que, além de causar o assoreamento dos rios, culmina na poluição dos cursos hídricos com arsênio (Rhodes, 2010; Sobreira, 2014; Santos, 2015). Muito embora a oxidação natural das rochas resulte na ampla disseminação do arsênio na biosfera, a presença de tal elemento na sua forma inorgânica em águas superficiais decorre, principalmente, do lançamento dos rejeitos da mineração aurífera nos seguimentos fluviais (Borba et al., 2004; Castilhos et al., 2020). Da ingestão de água com elevados teores de arsênio derivam uma miríade de possíveis complicações à saúde humana: câncer de pele, bexiga, rim e pulmão; diabetes; hipertensão arterial e distúrbios reprodutivos, dentre outras (WHO, 2018). Não por acaso, a Agência Internacional de Pesquisa em Câncer (IARC, 2021) e a Agência Norte Americana de Proteção Ambiental (EPA, 2021) classificaram o arsênio como elemento “cancerígeno para o ser humano". Portanto, o lançamento deste elemento traço nos cursos hídricos é um problema ambiental com repercussões deletérias na saúde pública devido à sua alta toxicidade (Castilhos et al., 2020).

Nos longevos tempos do ciclo do ouro, as cidades de Ouro Preto e Mariana, no estado de Minas Gerais, foram os maiores polos auríferos brasileiros. Não bastasse o fato destas cidades tricentenárias não tratarem adequadamente seus esgotos domésticos desde quando foram fundadas, o que já representa uma questão desafiadora no campo da gestão ambiental, nos últimos anos, diversas pesquisas comprovaram que as duas principais bacias hidrosedimentológicas e aquíferos subterrâneos da região apresentam níveis elevados de elementos traço, sobretudo de arsênio.

Borba et al. (2004) encontrou concentrações de arsênio impróprias para consumo humano nas águas subterrâneas da mina de ouro localizada em Passagem de Mariana, distrito do município de Mariana, desativada a 67 anos. Rhodes (2010) observou que o Rio Gualaxo do Norte apresentava maiores teores de arsênio em amostras de sedimentos nos pontos onde há atividade garimpeira. Costa et al. (2010) detectou concentrações de arsênio em amostras de sedimentos da bacia do Ribeirão do Carmo superiores às amostras da bacia do Rio Gualaxo do Norte, especialmente em áreas notabilizadas pela presença de garimpos de ouro. Santolin (2016) confirmou a contaminação de sedimentos na bacia do Ribeirão do Carmo e sua associação à atividade garimpeira em Monsenhor Horta. Os garimpos existentes em Monsenhor Horta, distrito do município de Mariana situado às margens do Ribeirão do Carmo, são uma herança histórica do ciclo do ouro (Amade \& Lima, 2009). A população de Monsenhor Horta, estimada em 1740 habitantes (IBGE, 2010), tem nos garimpos sua principal fonte de renda e emprego. Desde a sua fundação em 1697, a extração de ouro constitui as bases econômicas e mesmo culturais do distrito (Câmara et al., 1996; Amade \& Lima, 2009).

De acordo com dados publicizados pelo Instituto Mineiro de Gestão das Águas - IGAM, no que diz respeito à qualidade das águas, o Ribeirão do Carmo afigura-se como um dos cursos hídricos mais críticos da sub-bacia do Rio Piranga, bacia do Rio Doce. Neste sentido, visando contribuir com o planejamento e ações de governança do Comitê de Bacia Hidrográfica do Rio Piranga, objetivou-se com o presente trabalho avaliar o Índice de Qualidade das Águas (IQA) e a Contaminação por Tóxicos do Ribeirão do Carmo com base nos resultados obtidos pelo monitoramento de qualidade das águas empreendido pelo IGAM entre os anos de 2010 e 2019. 


\section{Metodologia}

\subsection{Caracterização da bacia hidrográfica do Ribeirão do Carmo}

Segundo o zoneamento ambiental produtivo do conjunto de sub-bacias hidrográficas do alto e médio baixo Ribeirão do Carmo (Minas Gerais, 2018), tal curso hídrico dispõe de área de drenagem 2.277,95 km² e nasce na Serra do Espinhaço a uma altitude de aproximadamente 1580 metros. Passa pela zona urbana da cidade de Ouro Preto identificado como Córrego Tripuí e Ribeirão do Funil, recebendo a denominação Ribeirão do Carmo ao adentrar o município de Mariana. Em seus 134 quilômetros de extensão, atravessa na direção leste-oeste a cidade de Mariana bem como três de seus distritos (a saber, Ribeirão do Carmo, Monsenhor Horta e Furquim, cujas manchas urbanas são demonstradas na Figura 1) e os municípios de Acaiaca e Barra Longa. $\mathrm{Na}$ foz do córrego Batalha, o Ribeirão do Carmo divide os municípios de Rio Doce e Ponte Nova e segue ao encontro do Rio Piranga. Na confluência com o Ribeirão do Carmo, o Rio Piranga passa a ser denominado Rio Doce (Minas Gerais, 2018).

Figura 1. Delimitação da bacia do Ribeirão do Carmo e indicação da estação amostral RD009.

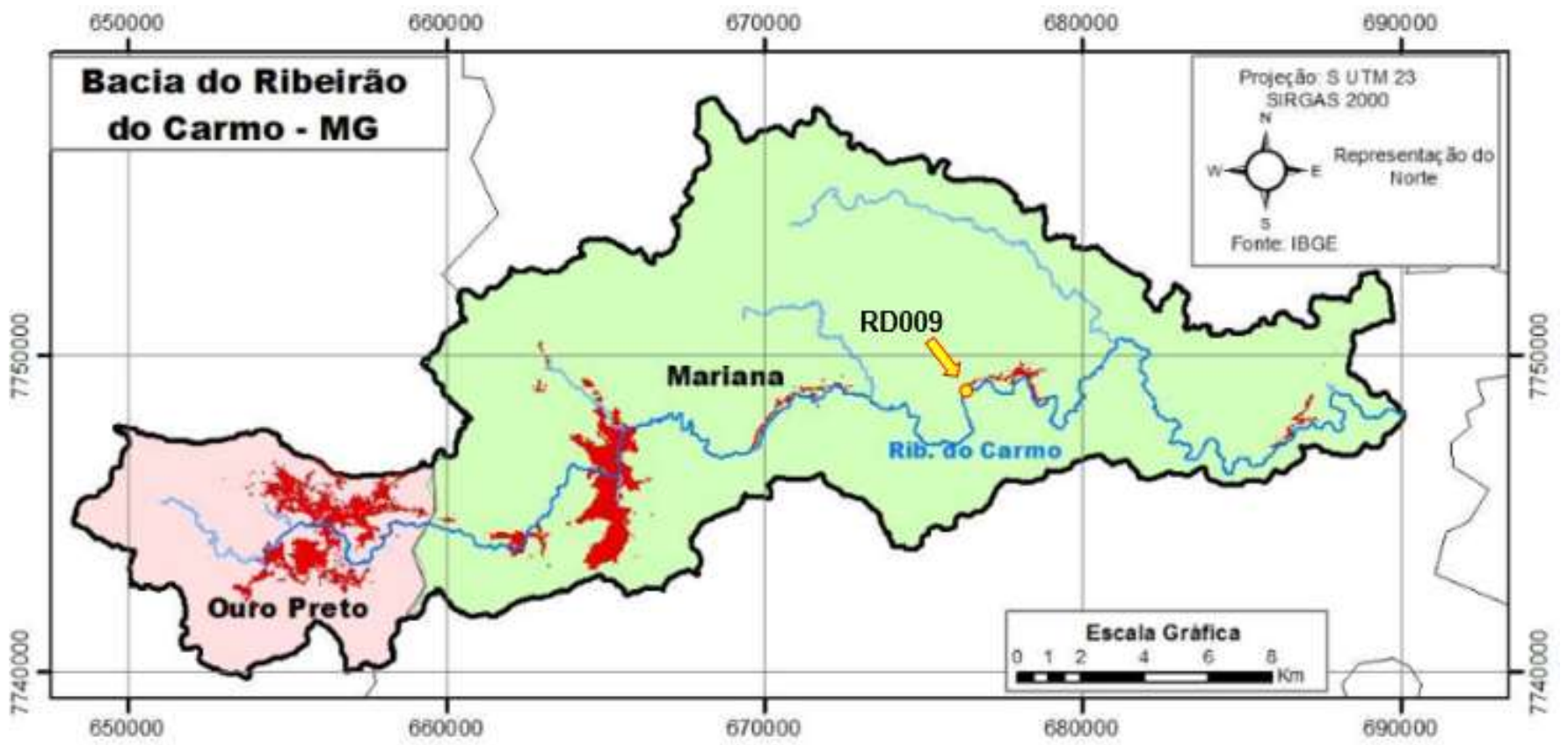

\section{Legenda:}

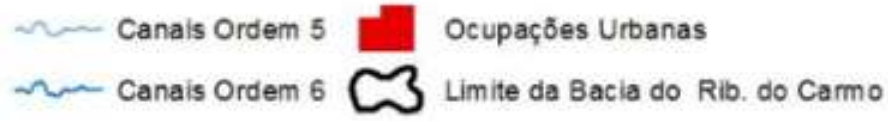

Como pode ser visualizado na Figura 2, a bacia do Ribeirão do Carmo é caracterizada por diferentes modalidades de usos e ocupações do solo. As unidades de cobertura vegetal secundária, uso mais prevalente, ocupavam 59,9\% da área da bacia em 2014. A título de comparação, em 1986, tais unidades perfaziam 147,1 km² enquanto, em 2014, 207,3 km², o que corresponde a um crescimento de 40,9\% desta tipologia de uso e ocupação do solo no período (Sobreira, 2018). A classe de campos, solos e pastagem, cuja maior parte é localizada nas imediações dos distritos de Ribeirão do Carmo, Monsenhor Horta e Furquim e na qual se concentram as atividades agropecuárias desenvolvidas na região, ocupavam 17,55\% da área da bacia em 2014. Em 1986, 
esta classe totalizava $127,4 \mathrm{~km}^{2}$ enquanto, em $2014,60,7 \mathrm{~km}^{2}$, o que representa uma redução de $52,4 \%$ deste tipo de uso do solo (Sobreira, 2018). Em 2014 as manchas urbanas ocupavam 4,86\% da área da bacia. Em 1986, as manchas urbanas ocupavam 9,6 $\mathrm{km}^{2}$ enquanto, em $2014,16,8 \mathrm{~km}^{2}$, o que configura uma expansão expressiva de $75,0 \%$ desta modalidade de uso do solo ao longo do Ribeirão do Carmo (Sobreira, 2018).

Figura 2. Mapa de uso e ocupação do solo da bacia do Ribeirão do Carmo em 2014.

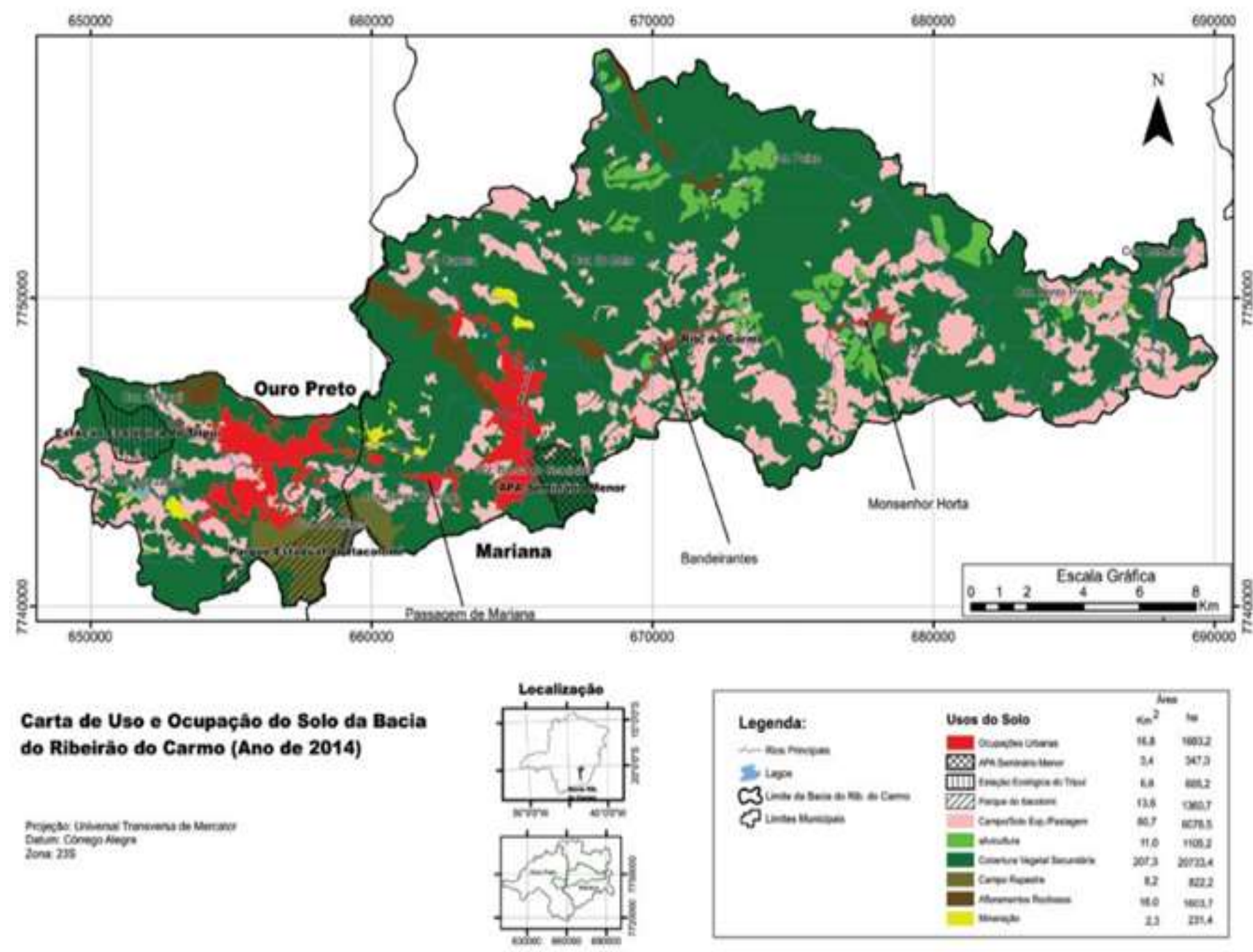

Fonte: Sobreira (2018

\subsection{Caracterização do uso e ocupação do solo do município de Mariana e do distrito de Monsenhor Horta}

A área territorial do município de Mariana é de 1.194,2 km² (IBGE, 2010). Segundo Souza (2004), as áreas dedicadas à pastagem, cobertura florestal e silvicultura compõem $84,79 \%$ do uso e ocupação do solo consoante demonstrado na Tabela 1 . No que diz respeito aos efluentes sanitários, a carga orgânica lançada no Ribeirão do Carmo pelo distrito sede é de $1.490,58 \mathrm{~kg}$ DBO5.dia ${ }^{-1}$ e não recebe tratamento adequado (Mariana, 2014).

A área territorial do distrito de Monsenhor Horta é de 111,97 km², o que corresponde a 9,38\% do território marianense (Souza, 2004). Embora inexistam dados oficiais ou trabalhos que caracterizem o uso e ocupação do solo do distrito, verificamse áreas dedicadas à silvicultura, agropecuária, exploração de gnaisse e aos garimpos clandestinos de ouro (Câmara et al., 1996; Souza, 2004; Amade \& Lima, 2009; Sobreira, 2018). Em apenas um dentre os diversos garimpos existentes em Monsenhor 
Horta, são empregados 174 trabalhadores (Amade \& Lima, 2009). Somente entre os anos de 2010 e 2019, a Polícia Militar do Estado de Minas Gerais realizou 51 autuações contra garimpos de ouro ilegais no município de Mariana, sendo que a maioria opera em Monsenhor Horta (Minas Gerais, 2021b). No que diz respeito aos efluentes sanitários, a carga orgânica lançada no Ribeirão do Carmo pelo distrito é de 31,42 kg DBO5.dia-1 e não recebe tratamento adequado (Mariana, 2014).

Tabela 1. Classes de uso e ocupação do solo no município de Mariana.

\begin{tabular}{ccc}
\hline USO DA TERRA & ÁREA $\left(\mathbf{K M}^{\mathbf{2}}\right)$ & PROPORÇÃo \\
\hline Pastagem & 428,0 & $35,84 \%$ \\
Cobertura Florestal & 368,0 & $30,90 \%$ \\
Silvicultura & 215,6 & $18,05 \%$ \\
Campos de Altitude & 51,1 & $4,28 \%$ \\
Áreas Legalmente Protegidas & 49,3 & $4,13 \%$ \\
Área de Domínio das Barragens (PCH's) & 40,7 & $3,41 \%$ \\
Mineração & 24,0 & $2,01 \%$ \\
Agrícolas & 16,4 & $1,38 \%$ \\
\hline Total & $\mathbf{1 . 1 9 4 , 2}$ & $\mathbf{1 0 0 \%}$ \\
\hline
\end{tabular}

Fonte: Adaptado de Souza (2004).

\subsection{Caracterização climática do município de Mariana}

O município de Mariana está situado na zona de clima tropical do tipo “Cwa” segundo a classificação de Koppën (Rubel \& Kotek, 2010), que indica clima temperado úmido com inverno seco e verão quente (Mariana, 2014). Como demonstrado na Tabela 2, considerando-se registros de precipitação da estação pluviométrica n 2043011 (denominada "FAZENDA PARAÍSO", localizada em Mariana) compreendidos entre os anos de 2010 e 2019 disponibilizados pela Agência Nacional de Águas (ANA) no portal Hidroweb, observa-se que a área de estudo exibe relevante amplitude pluviométrica. Deu-se no mês de dezembro a maior precipitação média mensal $(297,8 \mathrm{~mm})$ enquanto a menor, 7,0 mm, ocorreu em julho.

Tabela 2. Precipitação média mensal (mm) na estação pluviométrica no 2043011 entre os anos de 2010 e 2019.

\begin{tabular}{ccccccccccccc}
\hline \multicolumn{10}{c}{ Precipitação média mensal (mm) no posto 2043011 entre 2010 e 2019 } \\
\hline Mês & Jan & Fev & Mar & Abr & Mai & Jun & Jul & Ago & Set & Out & Nov & Dez \\
\hline Média & 178,9 & 109,0 & 208,2 & 88,9 & 47,8 & 21,3 & 7,0 & 14,3 & 44,4 & 83,7 & 205,4 & 297,8 \\
\hline
\end{tabular}

Fonte: Autores,

\subsection{Metodologia para avaliação do IQA e contaminação por tóxicos}

Visando a avaliação do IQA e contaminação por tóxicos do Ribeirão do Carmo no período compreendido entre os anos de 2010 e 2019, foram adotados os dados publicizados pelo IGAM no portal InfoHidro (Minas Gerais, 2021a) quanto às 39 campanhas de monitoramento de qualidade das águas empreendidas com periodicidade trimestral na estação amostral RD009, localizada no distrito de Monsenhor Horta, município de Mariana, conforme descrição apresentada na Tabela 3 e indicação constante na Figura 1. 
Research, Society and Development, v. 10, n. 10, e110101018680, 2021

(CC BY 4.0) | ISSN 2525-3409 | DOI: http://dx.doi.org/10.33448/rsd-v10i10.18680

Tabela 3. Descrição do ponto de monitoramento

\begin{tabular}{llccccc}
\hline Código & Localização & Lat. & Long. & Altitude & Período & Campanhas \\
\hline RD009 & $\begin{array}{l}\text { Ribeirão do Carmo } \\
\text { em Monsenhor }\end{array}$ & $-20,35$ & $-43,32$ & 651 & $2010-2019$ & 39 \\
& & & & & & \\
\hline
\end{tabular}

Fonte: Autores,

Para a avaliação do IQA (Eq. 1) foram adotados o conjunto de nove parâmetros considerados mais representativos para a caracterização da qualidade de água e seus respectivos pesos de acordo com sua importância relativa no cálculo do IQA: oxigênio dissolvido (peso 0,17); coliformes termotolerantes (peso 0,15); potencial hidrogeniônico (peso 0,12); demanda bioquímica de oxigênio (peso 0,10); fosfato total (peso 0,10); temperatura da água (peso 0,10); nitratos (peso 0,10); turbidez (peso 0,08) e sólidos totais (peso 0,08). O IQA foi calculado pelo produto ponderado dos pesos atribuídos a cada parâmetro de qualidade de água segundo metodologia apresentada pelo IGAM (2018b).

$$
\mathrm{IQA}=\prod_{\mathrm{i}=1}^{9} \mathrm{q}_{\mathrm{i}}^{\mathrm{wi}}
$$

Onde:

IQA= Índice de Qualidade de Água, variando de 0 a 100.

qi = qualidade do parâmetro i, obtida através da curva média específica de qualidade;

wi= peso atribuído ao parâmetro, em função de sua importância na qualidade entre 0 e 1.

Assim determinado, o IQA foi classificado de acordo com os critérios adotados pelo IGAM (2018b) demonstrados na Tabela 4.

Tabela 4. Classificação do IQA

\begin{tabular}{cc}
\hline Valor do IQA & Classes \\
\hline $90<\mathrm{IQA} \leq 100$ & Excelente \\
$70<\mathrm{IQA} \leq 90$ & Bom \\
$50<\mathrm{IQA} \leq 70$ & Médio \\
$25<\mathrm{IQA} \leq 50$ & Ruim \\
$\mathrm{IQA} \leq 25$ & Muito Ruim \\
\hline
\end{tabular}

Fonte: Adaptado de IGAM (2018b).

Conforme metodologia do IGAM (2018a) exposta na Tabela 5, a contaminação por tóxicos foi classificada em "Baixa", "Média" ou "Alta" a partir das concentrações observadas na estação amostral RD009 de quatorze parâmetros tóxicos: arsênio total, bário total, cádmio total, chumbo total, cianeto livre e cianeto total, cobre dissolvido, cromo total, fenóis totais, mercúrio total, nitrito, nitrato, nitrogênio amoniacal total e zinco total. 
Research, Society and Development, v. 10, n. 10, e110101018680, 2021

(CC BY 4.0) | ISSN 2525-3409 | DOI: http://dx.doi.org/10.33448/rsd-v10i10.18680

Tabela 5. Faixas de classificação do Índice de Contaminação por Tóxicos

\begin{tabular}{cc}
\hline Concentração & Classe de contaminação \\
\hline$=1,2 . P$ & Baixa \\
$1,2 . P<$ concentração $=2 . P$ & Média \\
Concentração $>2 . P$ & Alta \\
\hline
\end{tabular}

$\mathrm{P}=$ Limite definido pela Deliberação Normativa Conjunta COPAM/CERH-MG N. ${ }^{\circ}$ 1, de 05 de Maio de 2008.

Fonte: IGAM (2018a).

Na classe baixa as substâncias tóxicas apresentam concentrações iguais ou inferiores a 20\% dos limites; na classe média ocorrem concentrações entre $20 \%$ e $100 \%$ dos limites e na classe alta às concentrações são superiores a 100\% dos limites. A pior situação do conjunto de resultados define a faixa de contaminação. Neste caso, a contaminação na estação RD009 é classificada como alta quando um dos parâmetros apresenta valor acima de 100\% (o dobro da concentração limite) em pelo menos uma das campanhas do ano.

Visando identificar os parâmetros de qualidade de água que tiveram maior peso sobre o IQA obtido, empreendeu-se análise estatística descritiva dos dados através da aplicação de fórmulas de tendência central (média, máximo e mínimo) e medidas de dispersão (desvio padrão e coeficiente de variação) disponíveis no software Excel 2016. Por fim, para avaliação da evolução temporal ao longo do período adotado, representou-se graficamente, no software Excel 2016, a variação do IQA obtido considerando-se a sazonalidade climática (período seco e período chuvoso) assim como os parâmetros que mais influenciaram os resultados do IQA e da Contaminação por Tóxicos além dos seus respectivos limites de tolerância segundo os critérios da Deliberação Normativa COPAM-CERH n ${ }^{\circ}$ 01/2008 para a classe de enquadramento 2.

\section{Resultados e Discussão}

\subsection{Análise do IQA}

Na Tabela 6 são exibidos os valores mínimo, máximo e médio, o desvio padrão (DP) e o coeficiente de variação (CV) dos parâmetros de qualidade de água da estação amostral RD009 bem como do IQA no período em análise. O CV obtido para turbidez $(189,12 \%)$, coliformes termotolerantes $(180,74 \%)$ e fósforo $(68,58 \%)$ foram os mais elevados, o que demonstra a heterogeneidade dos resultados destes parâmetros. Sucessivamente, é preciso destacar que os valores médios e o DP de coliformes tolerantes (27691,27 NMP.100 $\left.\mathrm{mL}^{-1}\right)$ e fósforo total $\left(0,08 \mathrm{mg} \mathrm{P.L}^{-1}\right)$ foram superiores aos valores máximos permitidos pela Deliberação Normativa COPAM-CERH nº 01/2008.

Tabela 6. Estatística descritiva dos parâmetros de qualidade de água da estação amostral RD009 durante o período considerado

\begin{tabular}{cccccccccccc}
\hline & & \multicolumn{10}{c}{ Parâmetros } \\
\cline { 3 - 12 } Código & Estatística & T (C) & pH & TUR & DBO & OD & ST & P & N & CT & IQA \\
& & & & & & & & & & \\
& Mín. & 17,40 & 6,10 & 2,10 & 2,00 & 6,30 & 59,00 & 0,05 & 0,02 & 30,00 & 38,62 \\
\multirow{2}{*}{ RD009 } & Máx. & 31,50 & 7,60 & 357,00 & 9,10 & 9,00 & 387,00 & 0,45 & 3,82 & 92084,00 & 79,66 \\
& Média & 24,38 & 6,95 & 39,79 & 2,75 & 7,20 & 153,41 & 0,12 & 1,30 & 15320,94 & 62,66 \\
& DP & 3,33 & 0,35 & 75,24 & 1,33 & 0,68 & 88,63 & 0,08 & 0,78 & 27691,27 & 10,07 \\
& CV (\%) & 13,65 & 4,99 & 189,12 & 48,36 & 9,51 & $\mathbf{5 7 , 7 8}$ & $\mathbf{6 8 , 5 8}$ & $\mathbf{5 9 , 6 5}$ & 180,74 & 16,07 \\
\hline
\end{tabular}

Legenda: $\mathrm{T}=$ Temperatura, em ${ }^{\circ} \mathrm{C} ; \mathrm{pH}=$ Potencial hidrogeniônico; TUR $=$ Turbidez, UNT; $\mathrm{DBO}=$ Demanda Biológica de Oxigênio, em $\mathrm{mg} . \mathrm{L}^{-1} ; \mathrm{OD}=$ Oxigênio Dissolvido, em \%ODSat; $\mathrm{ST}=$ Sólidos Totais, em mg. $\mathrm{L}^{-1} ; \mathrm{P}=$ Fóforo Total, em mg P. $\mathrm{L}^{-1} ; \mathrm{N}=\mathrm{Nitrogênio} \mathrm{Total,}$ em mg. $\mathrm{L}^{-1} \mathrm{NO}_{3} ; \mathrm{CT}=$ Coliformes Termotolerantes, em NMP. $100 \mathrm{~mL}^{-1} ;$ IQA = Índice de Qualidade da Água.

Fonte: Autores, 
Research, Society and Development, v. 10, n. 10, e110101018680, 2021

(CC BY 4.0) | ISSN 2525-3409 | DOI: http://dx.doi.org/10.33448/rsd-v10i10.18680

O IQA médio da série histórica foi 62,66, valor classificado como "médio" pelo IGAM (2018b). Das 39 campanhas realizadas, 27 (69,2\%) receberam tal classificação. Como pode ser observado na Figura 3, o IQA da estação amostral RD009, no período adotado, caracteriza-se pela irregularidade e pela elevada amplitude de variação.

Figura 3. Evolução temporal do IQA da estação amostral RD009

Evolução temporal do IQA da estação amostral RD009

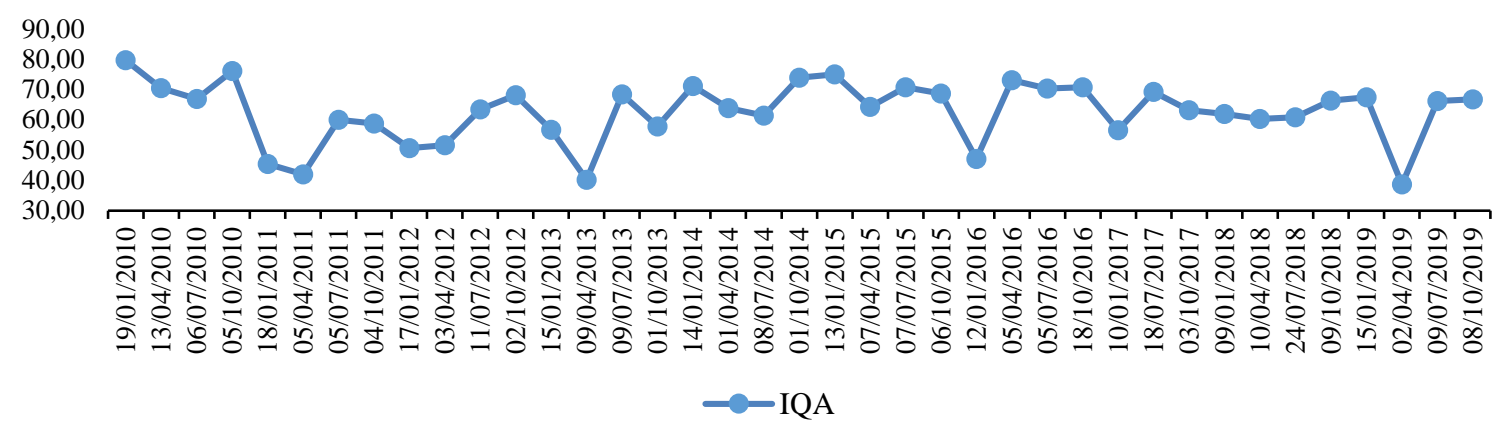

Fonte: Autores,

Com base nos critérios do IGAM (2018b), em 15,4\% das amostragens empreendidas o IQA foi classificado como “bom”, sendo que na primeira campanha de monitoramento (janeiro de 2010) atingiu-se o resultado de 79,66, maior da série histórica. Por outro lado, a campanha de abril de 2019 apresentou IQA de 38,62 (“ruim”), menor do período. Tal resultado está associado aos valores de DBO $\left(9,1 \mathrm{mgO}_{2} \cdot \mathrm{L}^{-1}\right)$, turbidez $(357,0 \mathrm{UNT})$, coliformes termotolerantes $\left(24196,0 \mathrm{NMP} .100 \mathrm{~mL}^{-1}\right)$ e fósforo $\left(0,45 \mathrm{mgP} . \mathrm{L}^{-1}\right)$ aferidos. Elevados teores de fósforo, turbidez e coliformes termotolerantes implicaram outros 3 resultados classificados como "ruim" nas amostragens de janeiro de 2011 (45,41), abril de 2011 (41,97) e abril de 2013 (40,23). Já em janeiro de $2012(50,63)$ e janeiro de $2016(47,12)$ o IQA “ruim” deriva dos altos valores de fósforo e coliformes termotolerantes. É pertinente destacar que estes dois últimos aludidos parâmetros excederam expressivamente os limites preconizados na Deliberação Normativa COPAM-CERH n n 01/2008 durante praticamente toda a série histórica como demonstram as Figuras 4 e 5 .

A exemplo do que demonstraram Johann (2010) e Silva (2013), os elevados teores de fósforo (Figura 4) provavelmente estão vinculados ao desenvolvimento de atividades agropecuárias na região que lançam efluentes de curral in natura no Ribeirão do Carmo. Já os níveis de coliformes termotolerantes (Figura 5) podem ser justificados pelo fato das cidades de Mariana (bem como o distrito de Ribeirão do Carmo) e Ouro Preto lançarem efluentes sanitários sem o devido tratamento no Ribeirão do Carmo, sendo esta uma condição análoga à investigada por Souza et al. (2017). Por fim, é possível que exista correlação entre os altos valores de turbidez (Figura 6) detectados nas amostragens realizadas em 18/01/2011, 05/04/2011, 09/04/2013 e 02/04/2019 com os níveis de coliformes termotolerantes, sólidos totais (que, apesar de não ter ultrapassado os limites estipulados na Deliberação Normativa COPAM-CERH n ${ }^{\circ}$ 01/2008, apresentou nas referidas amostragens 4 dos 5 maiores valores medidos), como corroboram as pesquisas de Siqueira et al. (2012) e Roberto et al. (2017) além do carreamento de partículas e substâncias tóxicas ao curso hídrico em tela pelo escoamento superficial oriundo às chuvas como afirma Blann et al. (2009), Kemerich et al. (2014) e Rodrigues et al. (2018). De acordo com dados disponibilizados pela Agência Nacional de Águas (ANA, 2021), no posto pluviométrico n 2043011, a precipitação registrada em 18 janeiro de 2011 foi 35,7 mm; em 05 de abril de 2011, 29,7 mm; em 09 de abril de 2013, 34,6 mm e; em 02 de abril de 2019, 23,3 mm. 
Research, Society and Development, v. 10, n. 10, e110101018680, 2021

(CC BY 4.0) | ISSN 2525-3409 | DOI: http://dx.doi.org/10.33448/rsd-v10i10.18680

Figura 4. Fósforo total - Evolução temporal na estação amostral RD009.

RD009 - Valores de Fósforo Total (mg P.L $\left.{ }^{-1}\right)$ x Limite DN COPAM-CERH n n $^{01 / 2008}$

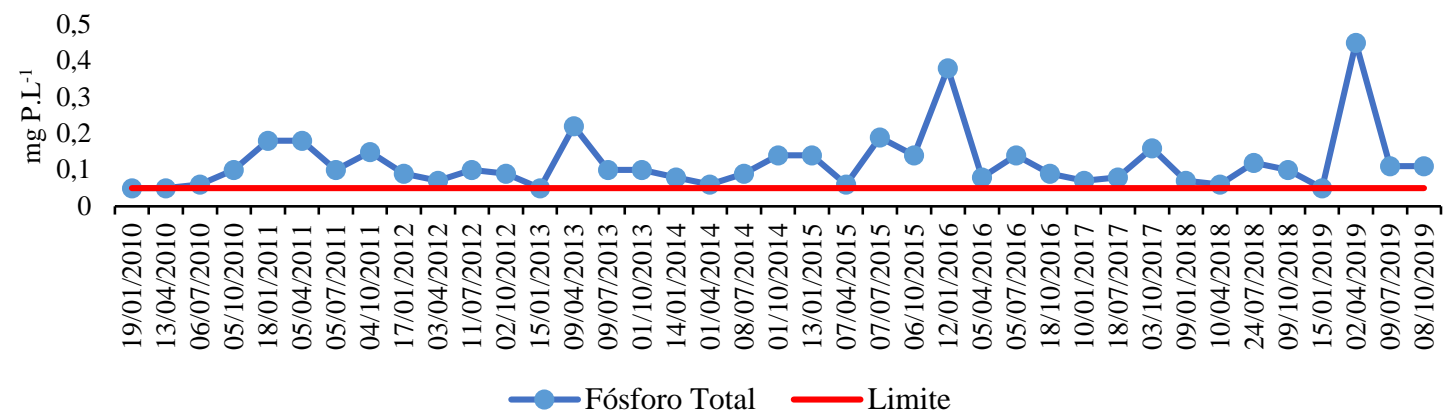

Fonte: Autores,

Figura 5. Coliformes termotolerantes - Evolução temporal na estação amostral RD009.

RD009 - Valores de Coliformes Termotolerantes (NMP.100 $\left.\mathrm{mL}^{-1}\right) \times$ Limite DN COPAMCERH n ${ }^{\circ}$ 01/2008

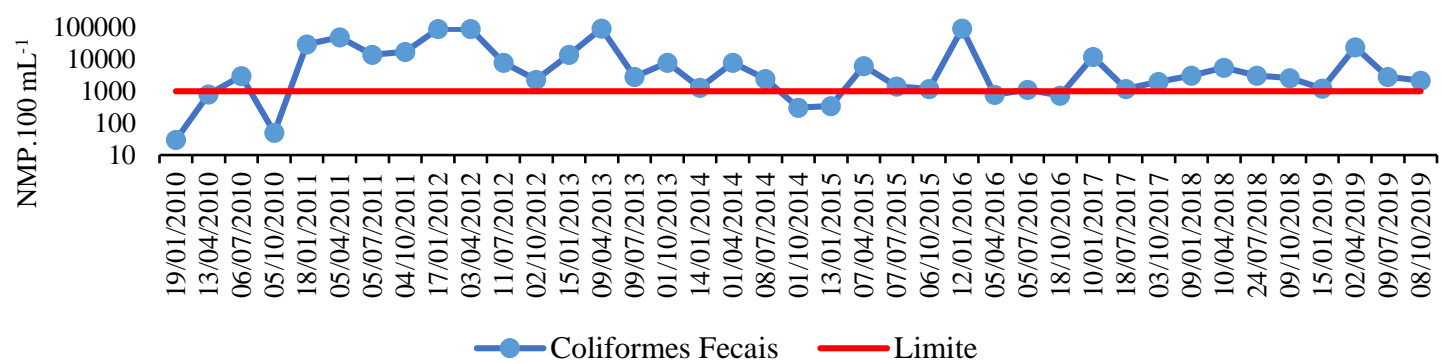

Fonte: Autores,

Figura 6. Turbidez - Evolução temporal na estação amostral RD009.

RD009 - Valores de Turbidez (UNT) x Limite DN COPAM-CERH nº1/2008 1000
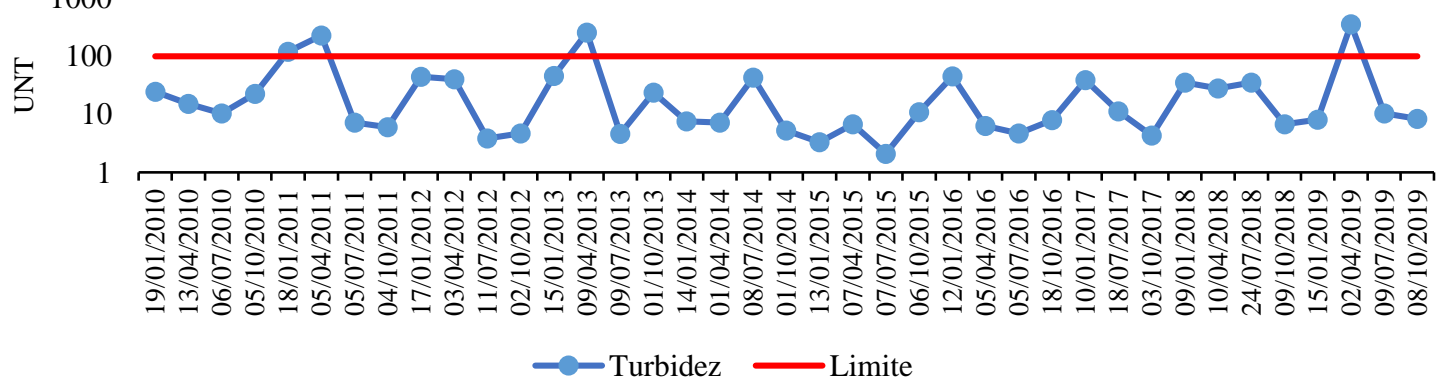

Fonte: Autores,

O IQA médio obtido nas campanhas do período chuvoso foi 64,10 ("médio") enquanto nas campanhas do período seco foi 61,14 ("médio"), queda relacionada à diminuição da vazão do rio típica dos meses secos do ano e à manutenção do aporte de 
carga orgânica lançada no corpo receptor, o que aumenta a concentração dos parâmetros e consequente piora do indicador. As Figuras 7 e 8 exibem a evolução temporal do IQA de acordo com a sazonalidade climática.

Figura 7. Evolução temporal do IQA da estação amostral RD009 - Período chuvoso.

Evolução temporal do IQA da estação amostral RD009 - Período chuvoso

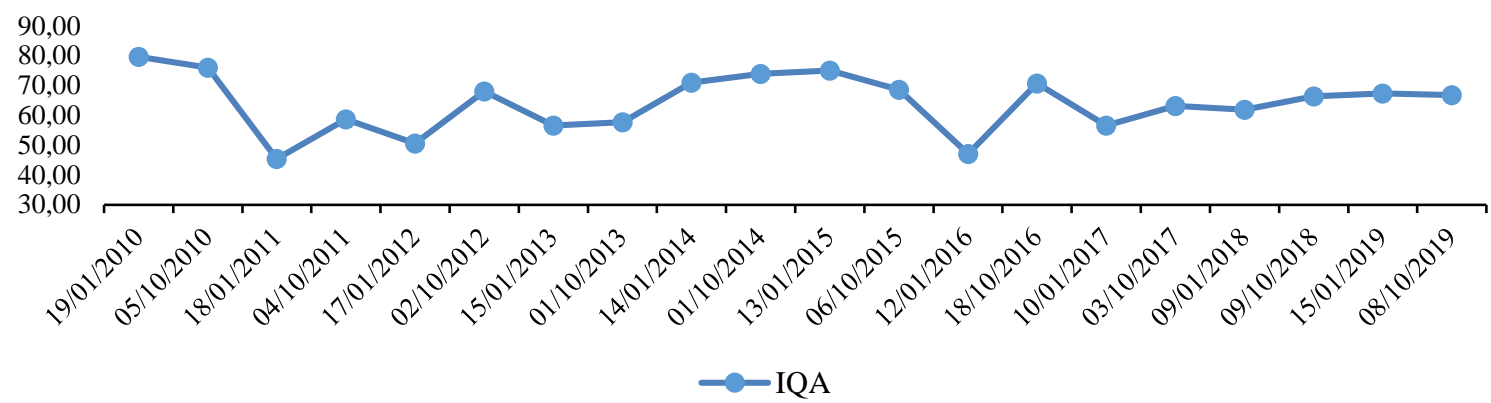

Fonte: Autores,

Figura 8. Evolução temporal do IQA da estação amostral RD009 - Período seco.

Evolução temporal do IQA da estação amostral RD009 - Período seco

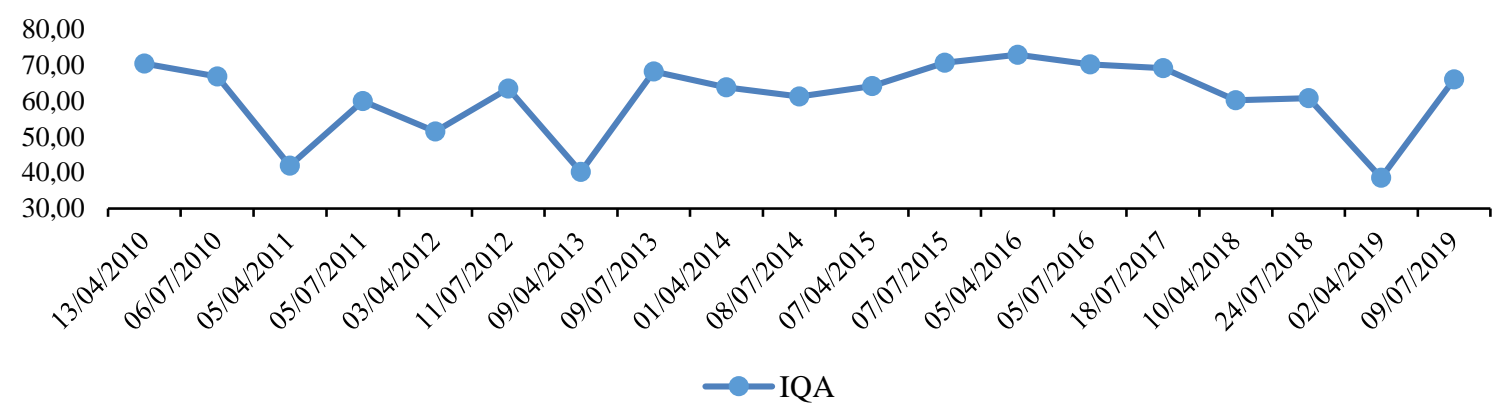

Fonte: Autores,

\subsection{Análise de contaminação por tóxicos}

A despeito dos parâmetros bário total, cádmio total, chumbo total, cianeto livre e cianeto total, cobre dissolvido, cromo total, fenóis totais, mercúrio total, nitrito, nitrato, nitrogênio amoniacal total e zinco total apresentarem concentrações classificadas como "baixa" na série histórica em comento, as concentrações de arsênio total foram elevadas e, por conseguinte, a contaminação por tóxicos no período é classificada como "alta" segundo os critérios do IGAM (2018a). Como demonstrado na Figura 9, excetuando-se o ano de 2014, classificado como "médio" em função dos resultados obtidos em julho (0,00261 mg.L$\left.{ }^{1}\right)$ e outubro (0,001 mg.L $\mathrm{L}^{-1}$, menor valor do período), nas demais amostragens as concentrações de arsênio total excederam o limite estabelecido para a classe 2 na Deliberação Normativa COPAM-CERH n ${ }^{\circ}$ 01/2008 (0,01 mg.L-1 $)$. Em abril de 2011 observou-se o maior valor no período considerado $\left(0,062 \mathrm{mg} \cdot \mathrm{L}^{-1}\right)$. Tendo em vista a presença de garimpos de ouro ilegais na região, é possível que exista correlação entre o desenvolvimento desta atividade e as altas concentrações de arsênio total identificadas, como sugeriu Santolin (2016). Cabe ainda considerar que a bacia hidrográfica do Ribeirão do Carmo é marcada pela significativa presença de arsênio em depósitos sedimentares (Costa et al., 2010). Assim, em observância a Blann et al. 
(2009), Kemerich et al. (2014) e Rodrigues et al. (2018), é possível que ocorra o transporte de tal elemento traço ao curso d’água pelo escoamento superficial provocado pelas precipitações pluviométricas.

Figura 9. Arsênio Total - Evolução temporal na estação amostral RD009.

\section{RD009 - Valores de Arsênio Total (mg.L-1 $\left.{ }^{-1}\right)$ Limite DN COPAM-CERH no $01 / 2008$}

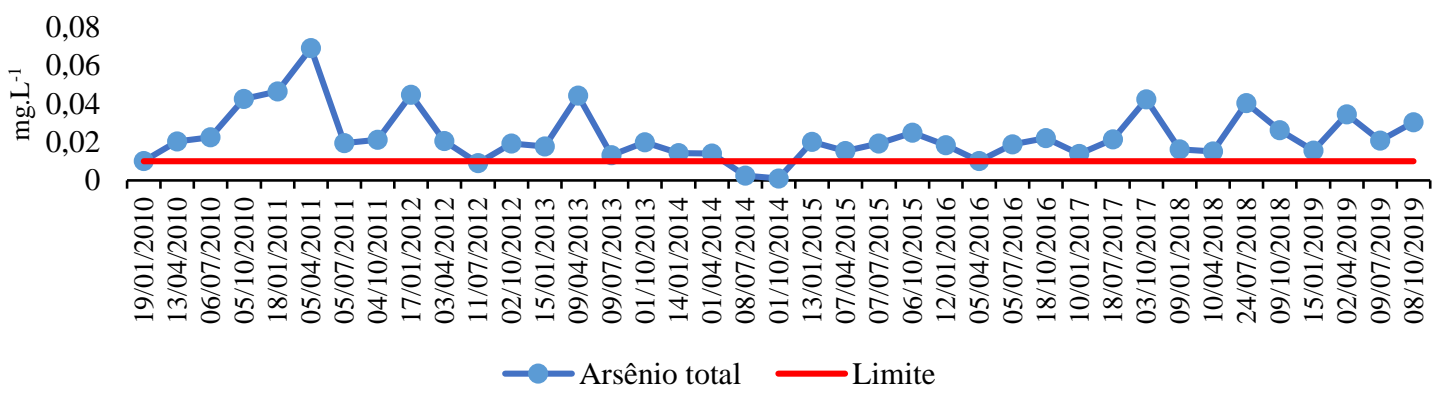

Fonte: Autores,

Adicionalmente, é salutar mencionar que no período de 2000 a 2009, malgrado o monitoramento de qualidade das águas superficiais empreendido pelo IGAM na estação amostral RD009 ter detectado a existência de arsênio total em níveis superiores aos limites estabelecidos pela Deliberação Normativa COPAM-CERH no 01/2008 em 68\% das amostragens, o Plano de Ação de Recursos Hídricos estipulado para a sub-bacia do Rio Piranga no ano de 2010 pelo Comitê de Bacia Hidrográfica do Rio Doce não teceu nenhuma consideração atinente aos garimpos de ouro existentes em Monsenhor Horta e suas possíveis vinculações com a presença de arsênio no Ribeirão do Carmo. Tendo em vista que a notável pesquisa interdisciplinar de Castilhos et al. (2020) demonstrou que a exposição humana ao arsênio é mais alta em regiões próximas à exploração de ouro, recomendase que os órgãos públicos de gestão e governança de recursos hídricos diligenciem mais profundamente sobre a problemática dos garimpos de ouro clandestinos comprovadamente existentes na região.

Por fim e não menos importante, observa-se na Figura 10 que as concentrações de mercúrio total, muito embora classificadas como "baixa" no período analisado, apresentaram valores iguais ao limite de tolerância estipulado na Deliberação Normativa COPAM-CERH n ${ }^{\circ}$ 01/2008 em todas as campanhas. Dado que o mercúrio é largamente utilizado pela atividade garimpeira para amalgamação das partículas auríferas extraídas e que as águas residuárias geradas neste processo são inadequadamente lançadas nos solos ou mesmo nos cursos hídricos, recomenda-se que os organismos de gestão e governança de bacias hidrográficas competentes articulem ações preventivas a fim de se evitar que as concentrações deste elemento traço atinjam valores capazes de causar as mesmas catástrofes ocorridas no Rio Tapajós, Estado do Pará, relatadas por Oliveira et al., (2015) e Basta \& Hacon (2020). 
Research, Society and Development, v. 10, n. 10, e110101018680, 2021

(CC BY 4.0) | ISSN 2525-3409 | DOI: http://dx.doi.org/10.33448/rsd-v10i10.18680

Figura 10. Mercúrio Total - Evolução temporal na estação amostral RD009.

\section{RD009 - Valores de Mercúrio Total $\left(\mathrm{mg.L}^{-1}\right)$ x Limite DN COPAM-CERH ${ }^{\circ}$ $01 / 2008$}

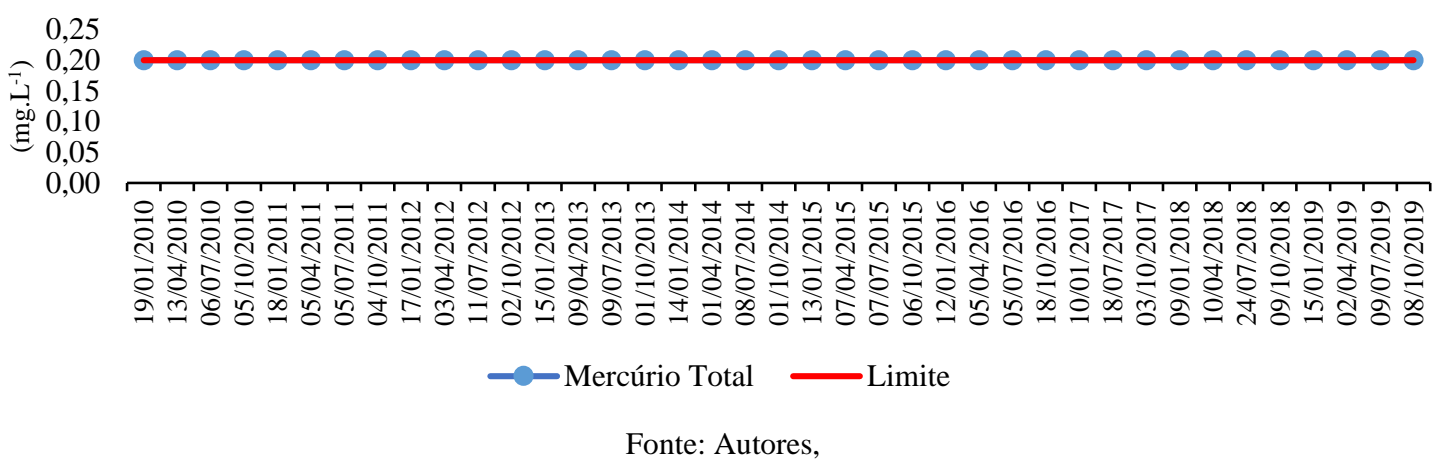

\section{Conclusão}

O IQA médio da série histórica foi 62,66, valor classificado como "médio". Das 39 campanhas, 6 (15,4\%) foram classificadas como "bom”, 27 (69,2\%) como “média" e outras $6(15,4 \%)$ como "ruim". O parâmetro coliformes termotolerantes ultrapassou o limite determinado na Deliberação Normativa COPAM-CERH nº 01/2008 em 82,05\% das campanhas enquanto o parâmetro fósforo total superou em $89,74 \%$ das amostragens os valores máximos permitidos na referida normativa. O lançamento de esgotos sanitários sem o devido tratamento assim como de efluentes de curral in natura no Ribeirão do Carmo se correlacionam com os resultados destes dois parâmetros. A contaminação por tóxicos é classificada como “alta” em função das elevadas concentrações de arsênio total detectadas em 94,87\% das amostragens. Das 39 campanhas, 37 identificaram a presença deste elemento traço em quantidades superiores aos limites determinados na Deliberação Normativa em epígrafe, sendo possível que garimpos clandestinos de ouro existentes no distrito de Monsenhor Horta contribuam com a presença de arsênio no Ribeirão do Carmo.

Este trabalho será encaminhado aos órgãos públicos de gestão e governança de recursos hídricos competentes para contribuição com a formulação de diretrizes e estratégias de melhoria da qualidade das águas do Ribeirão do Carmo.

\section{Agradecimentos}

À ex-professora Maria Angélica Fernandes (popularmente conhecida como “Tia Lete") que, na década de 1990, de forma pioneira, corajosa e mesmo arriscada, abriu os caminhos para a FEAM (Federação Estadual do Meio Ambiente de Minas Gerais) realizar as primeiras pesquisas relacionadas aos garimpos de ouro em Monsenhor Horta. Este trabalho, pois, é uma pequena fração das reflexões legadas pela Tia Lete em suas valorosas aulas de Geografia.

\section{Referências}

Amade, P. \& Lima, H. M. (2009). de. Desenvolvimento sustentável e garimpo - O caso do Garimpo do Engenho Podre em Mariana, Minas Gerais. Ouro Preto: Revista Escola de Minas, 62(2), 237-242.

ANA - Agência Nacional das Águas. (2021). HidroWeb. Disponível em: <http:// www.snirh.gov.br/hidroweb/serieshistoricas/>. Acesso em: 20 mai. 2021.

Araújo, P. L.; Hamburger, D. S.; Jesus, T. A. de.; Benassi, R. F. \& Cicco, V. de. (2018). Relação entre a qualidade da água e o uso do solo em microbacias do reservatório Billings, na Região Metropolitana de São Paulo - SP. Porto Alegre: Revista de Gestão de Água da América Latina, 15(2).

Basta, P. C. \& Hacon, S. S. (2020). Impacto do mercúrio na saúde do povo indígena Munduruku, na Bacia do Tapajós. Nota Técnica, WWF / FIOCRUZ. 7p. 
Research, Society and Development, v. 10, n. 10, e110101018680, 2021 (CC BY 4.0) | ISSN 2525-3409 | DOI: http://dx.doi.org/10.33448/rsd-v10i10.18680

Blann, K., Anderson, J., Sands, G. \& Vondracek, B. (2009). Potential implications of expanded agricultural sub-surface tile drainage for aquatic ecosystems in the Red River Basin. Cushing, USA.

Borba, R. P.; Figueiredi, B. R. \& Cavalcanti, J. A. (2004). Arsênio na água subterrânea em Ouro Preto e Mariana, Quadrilátero Ferrífero (MG). Ouro Preto: Revista Escola de Minas, 57(1), 45-51.

Brasil. Ministério da Saúde. Fundação Nacional de Saúde. Manual de Saneamento - 4. ed. - Brasília : Funasa, 2015.642 p. il.

Câmara, V. M.; Freitas Filhote, M. A. de; Lima, M. I. M.; Alheira, F. V.; Martins, M. S.; Dantas, T. O. \& Raggio Luiz, R. (1996). Metodologia para prevenir exposição ao mercúrio em adolescentes de garimpos de ouro em Mariana, Minas Gerais, Brasil. Rio de Janeiro: Caderno de Saúde Pública, 12(2), 149-158.

Castilhos, C. C.; Capitani, E. M.; Jesus, I. M.; Bidone, E. D.; Mello, W. Z.; Lima, M. O.; Faial, K. R. F.; Mataveli, L. R. V.; Arauz, L. J.; Silva, L. I. D.; Ferreira, A. P.; Távora, R. S. (2020). Avaliação da Contaminação Ambiental por Arsênio e Estudo Epidemiológico da Exposição Humana em Paracatu-MG - Brasil. Fronteiras: Journal of Social, Technological and Environmental Science. V.9, n.1, jan.-abr. 2020. P. 186-12.

Costa, A. T.; Nalini Jr, H. A.; Castro, P. T. A. \& Tatumi, S. H. (2010). Análise estratigráfica e distribuição do arsênio em depósitos sedimentares quaternários da porção sudeste do Quadrilátero Ferrífero, bacia do Ribeirão do Carmo, MG. Ouro Preto: Revista Escola de Minas, 63(4), 703-714.

EPA - Environmental Protection Agency - United State. (2021). Disponível em: < https://www.epa.gov/>. Acessado em: 13 de junho de 2021.

FAO - Food and Agriculture Organization of the United Nations. (2018). More people, more food, worse water? A global review of water pollution from agriculture. Rome, 2018.

IBGE - Instituto Brasileiro de Geografia e Estatística. (2010). IBGE: Cidades. Censo Demográfico. Disponível em: < https://cidades.ibge.gov.br/brasil/mg/mariana>. Acessado em: 11 de junho de 2021.

IARC - International Agency for Research on Cancer. (2021). Disponível em: < https://www.iarc.who.int/> Acessado em: 20 de junho de 2021.

IGAM - Instituto Mineiro de Gestão das Águas. (2018a). Contaminação por Tóxicos - CT. Disponível em: < http://portalinfohidro.igam.mg.gov.br/semcategoria/320-contaminacao-por-toxicos-ct> Acessado em: 15 de junho de 2021

IGAM - Instituto Mineiro de Gestão das Águas. (2018b). Índice de Qualidade Das Águas - IQA. Disponível em: < http://portalinfohidro.igam.mg.gov.br/semcategoria/319-indice-de-qualidade-das-aguas-iqa> Acessado em: 15 de junho de 2021

Johann, A. S. T. (2010). Desenvolvimento de tecnologia alternativa para tratamento de efluente da limpeza dos currais de gado leiteiro. Dissertação (Mestrado em Engenharia Química): Unioeste, Toledo. 109 f.

Kemerich, P. D. C.; Martins, S. R.; Kobiyama, M.; Flores, C. E. B.; Borba, F.; Fernandes, G. D.; Santi, A. L.; Cherubin, M. R. (2014). Infiltração e Escoamento Superficial Sob Diferentes Usos e Ocupação em uma Bacia Hidrográfica. Anuário do Instituto de Geociências, Vol. 37 - 2, p. $75-88$.

Mariana. (2014). Elaboração do Plano Municipal de Saneamento Básico (PMSB ) do Município de Mariana. Engecorps Engenharia S.A.. 358p. Disponível em: < https://www.mariana.mg.gov.br/uploads/prefeitura_mariana_2018/arquivos_veja_tambem/produto-8-relatorio-final-do-pmsb-de-mariana.pdf> Acessado em: 10 de junho de 2021.

Minas Gerais. (2008). Conselho Estadual de Política Ambiental / Conselho Estadual de Recursos Hídricos do Estado de Minas Gerais - COPAM/CERH-MG. Deliberação Normativa Conjunta COPAM / CERH-MG $n^{o} 01$, de 05 de maio de 2008 . Disponível em: < http://www.siam.mg.gov.br/sla/download.pdf?idNorma=8151> Acessado em: 10 de junho de 2021.

Minas Gerais. (2010). Plano de Ação de Recursos Hídricos da Unidade de Planejamento e Gestão DO1: PARH Piranga. Disponível em: < http://repositorioigam.meioambiente.mg.gov.br/handle/123456789/974 > Acessado em: 10 de junho de 2021.

Minas Gerais. (2021a). Instituto Mineiro de Gestão das Águas - IGAM. Séries Históricas de Monitoramento da Qualidade das Águas Superficiais do Estado de Minas Gerais. Disponível em: < http://www.repositorioigam.meioambiente.mg.gov.br/handle/123456789/405> Acessado em: 10 de junho de 2021.

Minas Gerais. (2018). Secretaria de Estado de Agricultura, Pecuária e Abastecimento de Minas Gerais. Zoneamento Ambiental Produtivo do Conjunto de SubBacias Hidrográficas do Alto e Médio Baixo Rio do Carmo. Disponível em: <https://drive.google.com/file/d/1Oo3ZMwhZdJPxzEgElectNDaArFqVYW6/view> Acessado em: 10 de junho de 2021.

Minas Gerais. (2021b). Secretaria de Estado de Justiça e Segurança Pública - SEJUSP/ Polícia Militar de Minas Gerais. Ofício $n^{\circ} 036 / 2021-3^{\circ} G P M A M B$ PM MAmb, de 01 de junho de 2021.

Oliveira, H. S. P.; França, S. C. A. \& Rocha, E. J. P. (2015). Atividades de mineração e avaliação de metais em água superficial, sedimento de fundo e peixes no Rio Tapajós. Amazônia em tempo, pp. 195-221. Em: VIEIRA, I. C. G.; JARDIM, M. A. G.; \& ROCHA, E. J. P. Amazônia em tempo: estudos climáticos e socioambientais. Belém: UFPA, Embrapa Amazônia Oriental. 462 p. 
Research, Society and Development, v. 10, n. 10, e110101018680, 2021 (CC BY 4.0) | ISSN 2525-3409 | DOI: http://dx.doi.org/10.33448/rsd-v10i10.18680

Rhodes, V. P. (2010). Distribuição de mercúrio e arsênio nos sedimentos da área afetada por garimpo de ouro - Rio Gualaxo do Norte, Mariana, Minas Gerais, $M G$. Dissertação Mestrado, UFOP. 93p.

Roberto, M. C.; Guimarães, A. P. M.; Ribeiro, J. L.; Carvalho, A. V. de; Neres, J. C. I. \& Cerqueira, F. B. (2017). Avaliação do ph, turbidez e análise microbiológica da água do Córrego Guará Velho em Guaraí, Estado do Tocantins. Revista Desafios, 4(4).

Rodrigues, R. S. S.; Bittencourt, G. M.; Fernandes, L. L. (2017). Escoamento Superficial em uma Pequena Bacia Hidrográfica Rural da Amazônia. Revista Brasileira de Cartografia, Vol. 79, n. 2, abril/junho, p. 605-628.

Rubel, F.; Kottek, M. (2010) Observed and projected climate shifts 1901-2100 depicted by world maps of the Köppen-Geiger climate classification. Meteorologische Zeitschrift, v.19, n.2, p.135-141.

Santolin, C. V. A. (2016). Quantificação e avaliação ambiental da contaminação por metais e arsênio em sedimentos da Bacia do Rio Doce - MG.Tese de Doutorado, UFMG. 157p.

Santos, M. C. B. (2015). Avaliação da contaminação por arsênio em solos, sedimentos e águas fluviais na região da mina de ouro “Morro do Ouro”, Paracatu$M G$. Niterói: UFF, Dissertação de Mestrado. 160 p.

Silva, E. M. (2013). Sistemas naturais para tratamento de resíduos líquidos de bovinocultura de leite. Campinas: UNICAMP, Tese de doutorado. 168 p.

Siqueira, G. W.; Aprile, F. \& Miguéis, A. M. (2012). Diagnóstico da qualidade da água do rio Parauapebas (Pará - Brasil). ACTA Amazônica, 42(3),413-422.

Sobreira, F. (2014). Mineração do ouro no período colonial: alterações paisagísticas antrópicas na serra de Ouro Preto, Minas Gerais. Quaternary and Environmental Geosciences, 05(1), 55-65.

Souza, L. A. (2004). Diagnóstico do meio físico como contribuição ao ordenamento territorial do município de Mariana (MG). Dissertação de Mestrado: UFOP. 182p.

Souza, Q. S.; Machado, C. M. S.; Moura, L. O. G. \& Lima, F. S. (2017) Análise de coliformes totais e termotolerantes-fecais em diferentes pontos da Sub-Bacia do Rio Poxim-Sergipe, Brasil. Agroforestalis News, 2(2).

Souza, L. A. \& Sobreira, F. G. (2018). Atlas Geoambiental: Bacia do Ribeirão do Carmo. Viçosa, MG: Asa Pequena. 84p.

WHO - World Health Organization. (2018). Arsenic. Disponível em: < https://www.who.int/news-room/fact-sheets/detail/arsenic> Acessado em: 9 de junho de 2021. 